\title{
Smartphone Application for Enhanced Indoor Health Environments
}

\author{
Gonçalo M. S. Marques ${ }^{1}$, Rui Pitarma ${ }^{1 *}$ \\ ${ }^{1}$ Polytechnic Institute of Guarda - Unit for Inland Development, Portugal \\ *Corresponding Author: rpitarma@ipg.pt \\ Citation: M. S. Marques, G., Pitarma, R. (2016) Smartphone Application for Enhanced Indoor Health \\ Environments, Journal of Information Systems Engineering \& Management, 1:4 (2016), 49.
}

doi: http://dx.doi.org/10.20897/lectito.201649

Received: June 17, 2016; Accepted: October 24, 2016; Published: November 7, 2016

\begin{abstract}
The reduced air quality inside buildings is undoubtedly a key factor to be controlled to ensure the health and comfort of the occupants as people usually spend more than $90 \%$ of their time in indoor environments. This paper aims to present $\mathrm{iAQ}$ an automatic system that is capable to monitoring indoor air quality and send real time notifications to provide active coaching for occupants in order to improve indoor air quality. iAQ is a cost effective solution for indoor air quality monitoring and provide monitoring data access by a web portal and a mobile application. This system is equipped is a five cost effective sensors for data acquisition of environmental parameters (air temperature, humidity, carbon monoxide, carbon dioxide and luminosity). Other sensors can be added to monitor specific pollutants. iAQ assumes increased interest due to the use of low cost and open source technologies. In fact, iAQ system has advantages in terms of simplicity of installation and configuration, through the use of wireless technology.
\end{abstract}

Keywords: Indoor air quality, indoor environment, air quality monitoring, wireless sensor network, ZigBee, Arduino, gas sensors, smart cities

\section{INTRODUCTION}

The indoor environments are characterized by several polluting sources. Thus, the indoor air quality (iAQ) is recognized as an important factor to be controlled to ensure and improve the health and comfort of the occupants. If we consider that today the massive majority of people spend more than $90 \%$ of their time in artificial environments (Spengler JD Et al 1983) is extremely important to reminder that health problems and diseases associated with poor indoor air quality can negatively affect productivity. According to the Environmental Protection Agency of the United States (USEPA, 2016), human exposure to indoor air pollutants in buildings may be 2 to 5 times, sporadically more than 100 times higher than outdoor pollution. Indoor environments accumulate the concentration of pollutants derivative from building materials, equipment, and also from daily activities of occupants (Mukhopadhyay, K Et al., 2016). In fact, indoor air pollutants were ranked among the top five environmental risks to public health (Fig.1). 


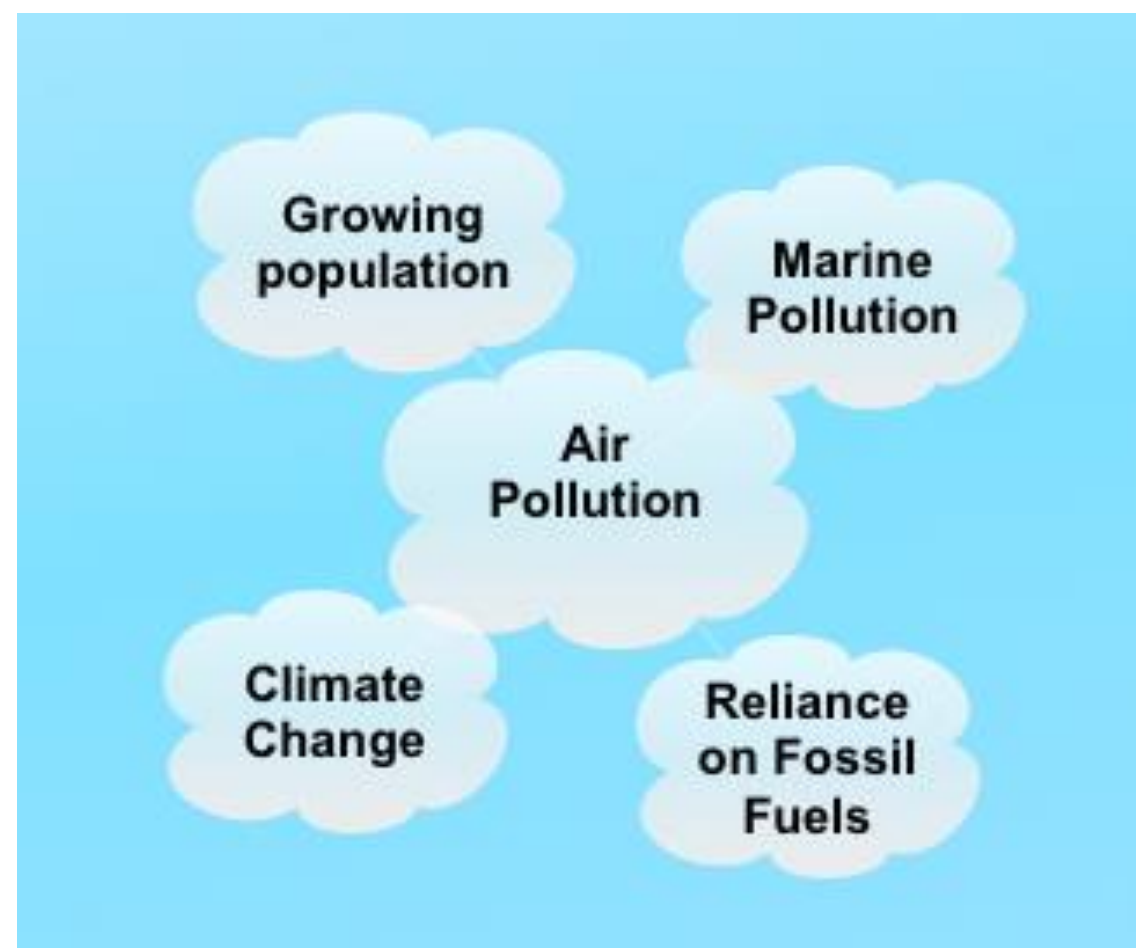

Figure 1. Top 5 Environmental Issues

Ventilation can be used in buildings to create thermally comfortable environments with decent air quality by regulating the indoor air parameters such as air temperature, relative humidity, air velocity and the concentrations of chemical species. A study on the computer simulation of the dispersion of a pollutant within a ventilated room with presentation of various numerical predictions is proposed by (Pitarma, R Et al., 2016).

Thus is evident that an indoor air quality monitoring system helps not only the detection but also the improving of air indoor air quality. Indeed, the local assessment and the chemical concentration distribution is significant for safety and occupant protection contributing to effectively control the temperature, ventilation and air conditioning (HVAC) in order to increase energy efficiency (De Vito, S Et al., 2011). In fact, the measurement of indoor air quality in buildings provides a continuous flow of information for continuous control and could also allowing the construction of automation systems and decision support platforms (Preethichandra, D, 2013). However, the currently available monitoring systems are expensive and only allow the collection of random samples. Recently, several new systems have been developed to monitor environmental parameters, always with the aim of enhance the efficiency and the indoor air quality (Yu, T Et al., 2015). In fact, the availability of inexpensive processors, low power, small size, in wireless communication technologies, sensors and actuators integrated in a single chip, allow the use of informatics to interact with the physical world in various applications such as the control of air quality (Al-Haija, Q Et al., 2013). A monitoring system for indoor air quality using wireless communication technology that provides real-time information for assistance to life is proposed by (Preethichandra, D., 2013). The proposed system has carbon dioxide, carbon monoxide, methane and propane sensors. Another wireless sensor network for air quality monitoring has been proposed in (Yu, T. Et al.,2013), it also incorporates ZigBee technology for wireless communication and humidity, temperature and carbon dioxide data monitoring.

This study describes the iAQ system, developed by the authors, which aims to ensure, independently and accurately monitoring, simultaneously, the indoor air quality in different rooms of a building. It consists of a monitoring of air quality using wireless communication technology system developed using Arduino and XBee technology that allows the storage and availability of monitoring data in real time through a web portal and mobile application for Android devices. The mobile application was created considering the fundamental role that the smartphone performances in world society, allowing to connect this system in constant contact with the end user. In fact, 56\% of adults in the US population are now carrying smartphones (Smith, A. Et al., 2013). In the Netherlands, $70 \%$ of the population and over $90 \%$ of teenagers own a smartphone (Rahmati, A. Et al., 2013). Moreover, one recent study, proposed in (Alexander Deursen Et al.,2015) conclude that the use of smartphones is performed, on average, 86 minutes per day. These arguments underline the importance of mobile devices in today's society. Another study, proposed by (Hintze, D. Et al., 2014) points out that the different patterns of use can be applied to different locations for each user, and smartphones are used considerably, even when users are close to computers, which contributes also to the motivation creation in this study of a mobile application. 
The system collects five environmental parameters (air temperature, humidity, carbon monoxide, carbon dioxide and luminosity) from different locations simultaneously. Other sensors can be added to monitor specific pollutants. Currently, the preliminary laboratory tests were used only two remote modules.

\section{TECHNICAL SOLUTION}

\section{A. Implementation}

The iAQ system is an automatic system for the indoor air quality monitoring that allows the end user, in particular the building manager to access in real time to a wide variety of environmental parameters such as air temperature, relative humidity, carbon monoxide (CO), carbon dioxide (CO2) and luminosity. Other sensors for specific pollutants can be added.

The iAQ system consists of a sensor network, the sensor node is called iAQ sensor and the central hub or node is denominated iAQ Gateway. The parameters are monitored using the iAQ sensor that collects air quality information and forwards it to the iAQ Gateway system which sends it using the web services developed in PHP to MySQL database.

The user can access the data from the web portal called for iAQ Web developed in PHP. Through iAQ Web, the user can see all information about environmental parameters. Monitoring data are shown as numerical values or as graphs. The iAQ web also allows the end user to maintain the history of indoor air quality for further analysis. Providing a history of air quality, the system helps the user to analyse precise and detailed the indoor air quality behaviour. In fact, the functionality cited above assumes huge importance in deciding on possible interventions to be carried out in the building in order to improve air quality. The Web iAQ is equipped with a powerful notification manager that allows the user to receive notifications when a particular parameter exceeds the maximum value.

In order to allow a quick, simple, intuitive and real-time access a mobile application was also been created with Android support, denominated for iAQ Mobile, described in detail in section C.

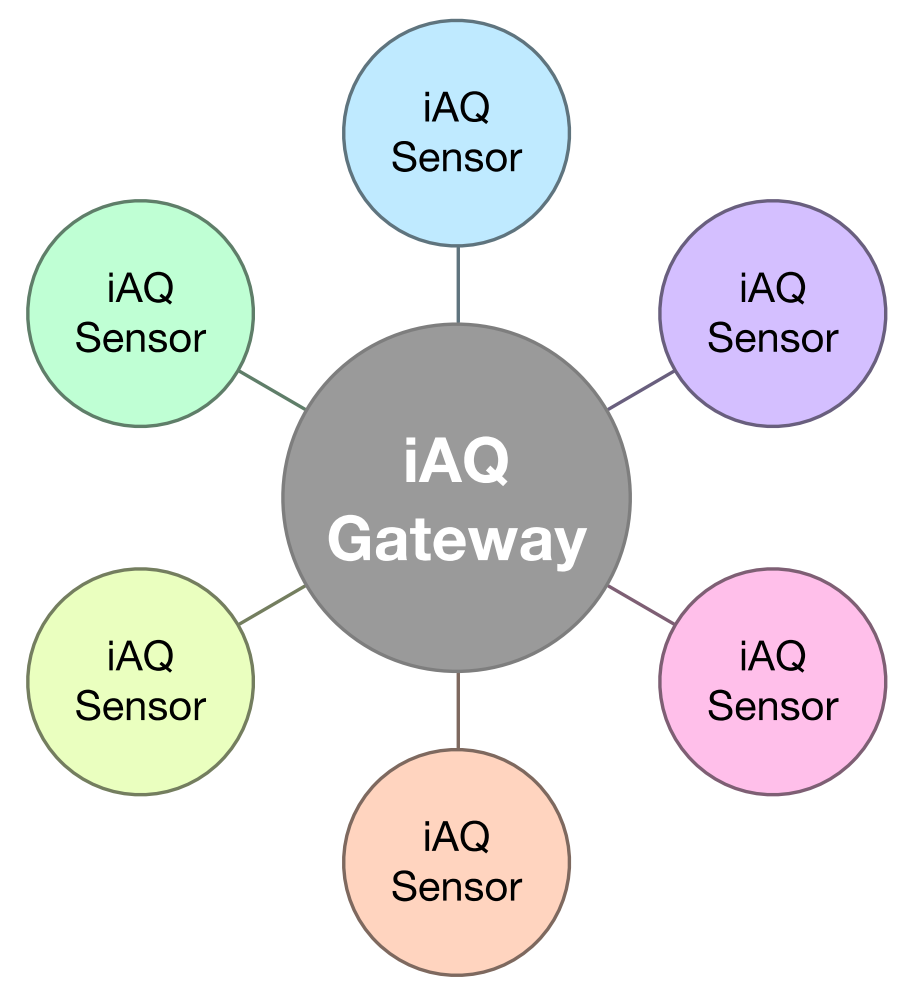

Figure 2. WSN Network

\section{B. Architecture of the sensor network}

The wireless communication is implemented using XBee module, which uses the IEEE 802.15.4 network protocol, and ZigBee Radio protocol (IEEE, 2016). The IEEE 802.15.4 standard specifies the access control layers for wireless communication in private areas. ZigBee is a wireless communication technology, low cost and low 
power, based on mesh networking standard built on 802.15.4 standard (ZigBee Alliance, 2016; Digi International, 2016).

Data collected from iAQ sensor are transferred to the iAQ Gateway using XBee wireless communication technology (Fig.2). The XBee modules operate within the $2.4 \mathrm{GHz}$ frequency band and have a capacity range in the open up to 4,000 feet $(1,200 \mathrm{~m})$ with RF data transfer rates of 250,000 bps. These modules use the IEEE 802.15.4 networking protocol for fast communication in point-to-multipoint or peer-to-peer networks. The XBee modules are designed for high performance applications that require low latency and predictable communication time. They are also ideal for applications where a low power consumption and low cost is required. The XBeePRO modules are amplified versions of the XBee modules for more demanding applications and a greater range (Ramya, V Et al., 2012).

\section{C. iAQ Mobile - mobile application for Android devices}

As already described, considering the importance of the smartphone's role in global society, a mobile application was created for Android devices. This application has authentication mechanisms and data protection. Before data access, the user must be authenticated by the username and the corresponding password.

After authentication, the iAQ Mobile allows the end user to view in detail the iAQ system data and receive notifications when a certain parameter exceeds the minimum or maximum values. AAQ Mobile is schematically illustrated in the Figure 3. This application was designed with the goal of providing quick and easy access to the iAQ system, in order to allow the end user to preserve with you all the relevant information collected by the iAQ system.

This mobile application was developed for the Android system using Android Studio IDE and has the API 15: Android 4.0.3 Ice Cream Sandwich as minimum development requirements, in order to be compatible with 96.2\% of active devices on Google Play Store (information collected on 22.01.2016).

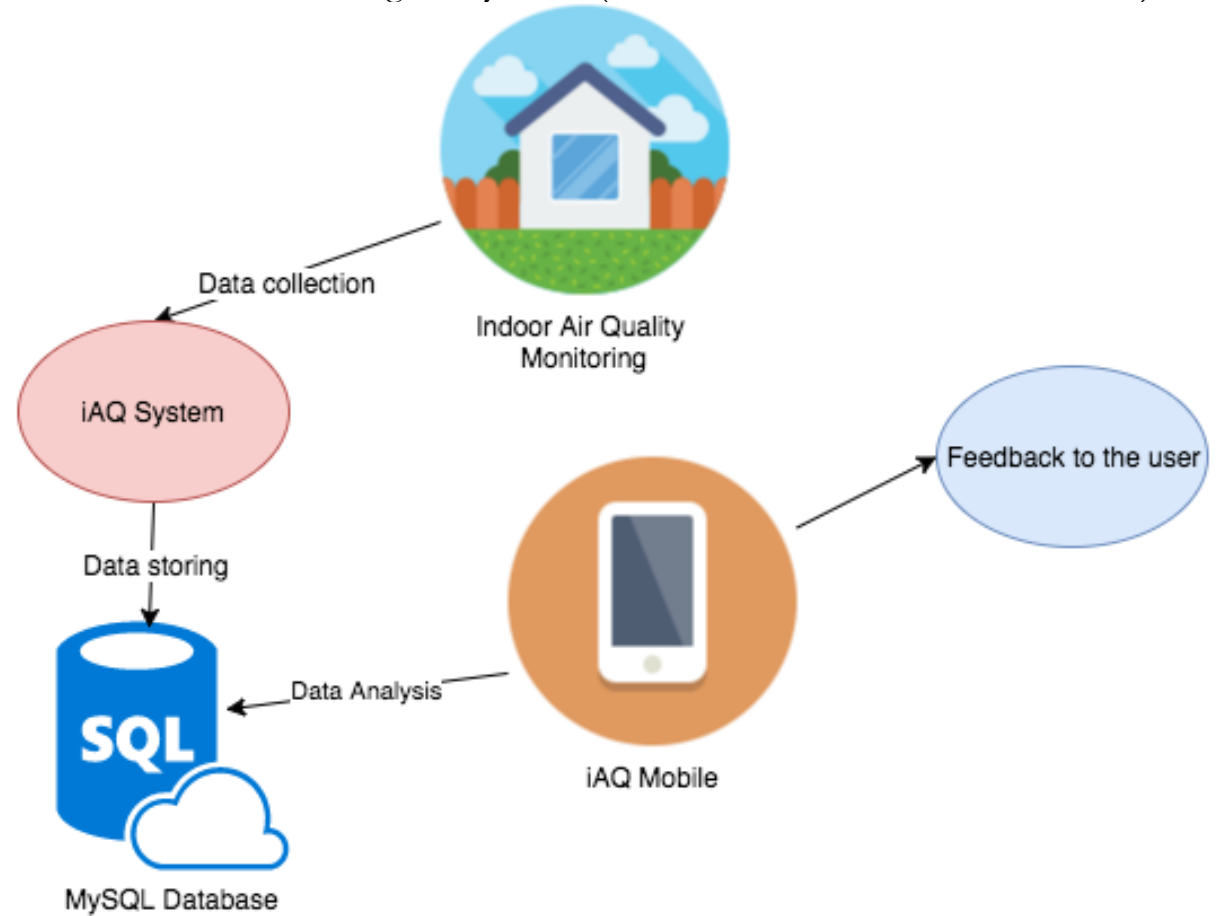

Figure 3. Application iAQ Android Mobile

\section{Hardware and system architecture}

The iAQ system consists in one or more iAQ sensor's modules used to collect and transport air quality data monitoring in different environments where they are installed. The iAQ sensor sends the data to the iAQ Gateway, which is connected to the Internet via an Ethernet Shield Arduino, to enable data storage in a database in realtime. Therefore, is possible to build a modular system that can control one or more indoor spaces simultaneously (Fig.4). 


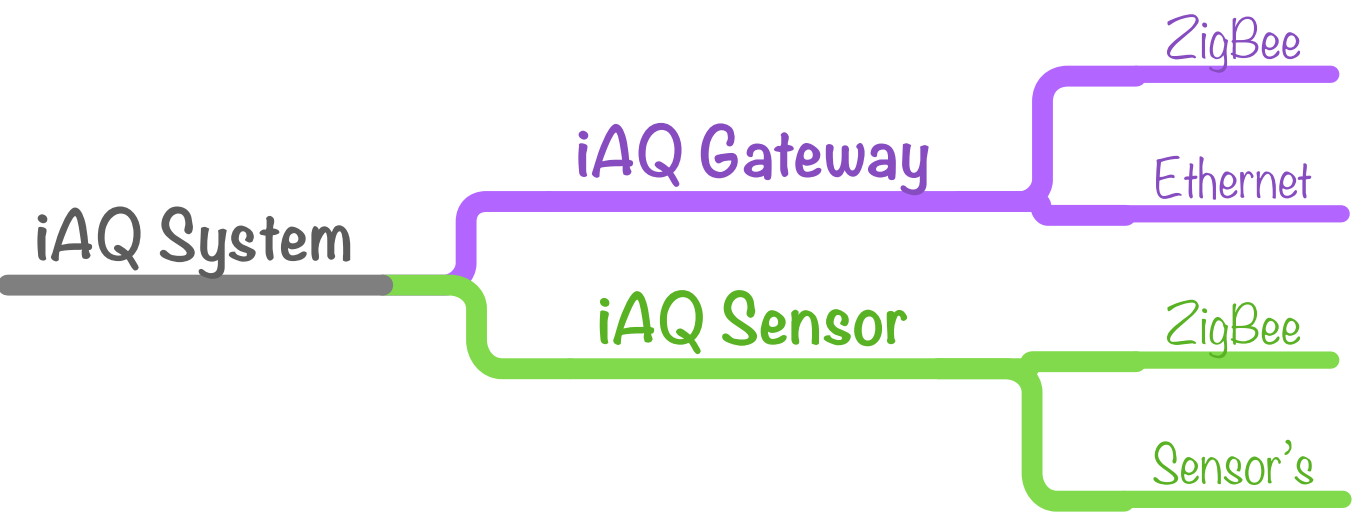

Figure 4. iAQ System

The iAQ sensor is developed using the Arduino MEGA embedded system, an open source platform that incorporates an Atmel AVR microcontroller (Michael, M Et al, 2012; Arduino, 2016). In order to allow communication between the iAQ Sensor and iAQ Gateway ZigBee technology is incorporated by using the XBee modules.

The AAQ sensor is equipped with multiple sensors, a processing unit (Arduino MEGA) and a wireless communication unit that allows mesh communication. Currently, the iAQ sensor is equipped with five sensors (Fig. 5): air temperature, relative humidity, carbon monoxide (CO), carbon dioxide (CO2) and light.

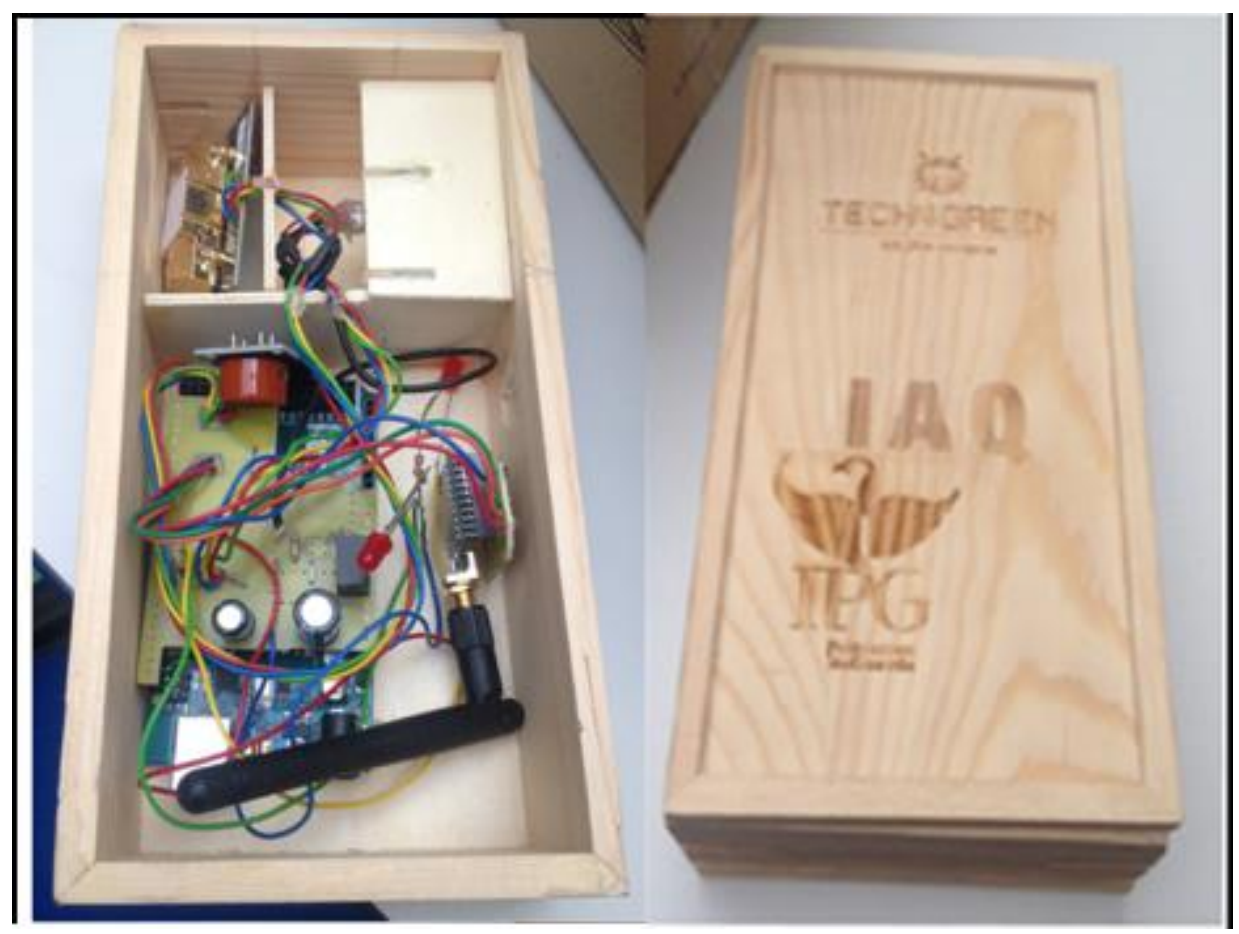

Figure 5. iAQ Sensor Hardware

A brief description of the sensors used is presented below.

- SHT10 - is a temperature and humidity sensor, low power, stable and fully calibrated (Sensirion, 2016); Measuring range: $0-100 \%$ (humidity) $-40 \sim 120{ }^{\circ} \mathrm{C}$ (temperature), accuracy: $\pm 4.5 \%$ (humidity), $\pm 0.5^{\circ} \mathrm{C}$ (temperature) and the response time is $<30 \mathrm{sec}$.

- MQ7 Sensor - is a high-sensitivity CO sensor (carbon monoxide) with various features (Sparkfun, 2016): high sensitivity, fast response, wide range of detection (20 to $2000 \mathrm{ppm}$ ), stable performance, long service life and requires calibration manual.

- T6615 CO2 Sensor - Sensor is a CO2 (carbon dioxide), low power, with a suitable performance, designed for HVAC purposes. Has the following main specifications (Telaire,2016) - Measuring range: 0-5,000ppm, 
precision: $\pm 50 \mathrm{ppm} \pm 3 \%$ of reading, response time: 2 minutes and features automatic calibration (every 24 hours).

- LDR $5 \mathrm{~mm}$ - is a sensor that allows luminosity monitoring. It is basically a resistor that changes its value (in ohms) depending on the amount of light (Robocore, 2016). It is a low-cost sensor, however, is inaccurate and should not be used to try to determine the brightness levels in absolute values, since it does not have sufficient accuracy. Thus, it is used to determine basic brightness changes. Resistance Range: $200 \mathrm{~K}$ ohm (dark) to $10 \mathrm{~K} \mathrm{ohm} \mathrm{(brightness} \mathrm{-} 10$ lux) sensitivity. Sensitivity range: CDS cells respond to light of wave length $400 \mathrm{~nm}$ (violet) and $600 \mathrm{~nm}$ (orange), reaching a maximum at about $520 \mathrm{~nm}$ (green).

\section{SOFTWARE}

The firmware iAQ sensor iAQ and Gateway was implemented using the language of the Arduino platform in the IDE Arduino, language belongs to the C programming family. The iAQ Web was developed in PHP and MySQL database (Fig.6). The web services that allow data collection are also built in PHP (Wang, M., Et al.,2013). The iAQ Mobile was developed for the Android system using IDE Android Studio using the Java language.

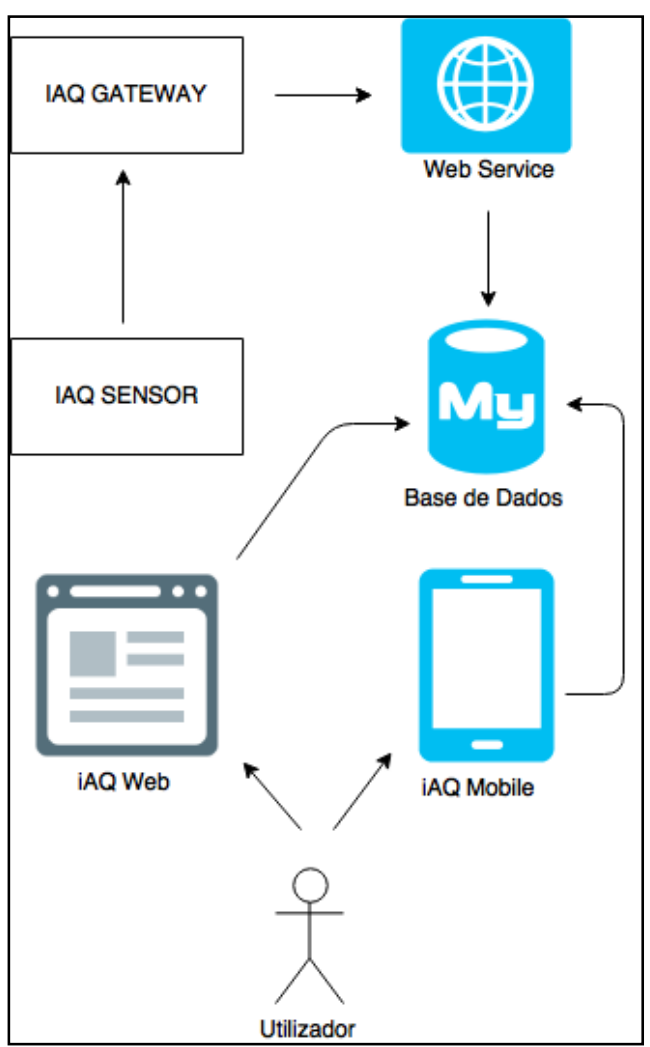

Figure 6. System Architecture

\section{DISCUSSION AND RESULTS}

The iAQ Web allows the visualization indoor air quality in the form of numerical values or as graphs. A sample of experimental data for a selected environment is illustrated in Figures 7 to 9. As examples were chosen relative humidity graphs (Figure. 7), air temperature (Figure. 8) and CO2 (Figure. 9). 


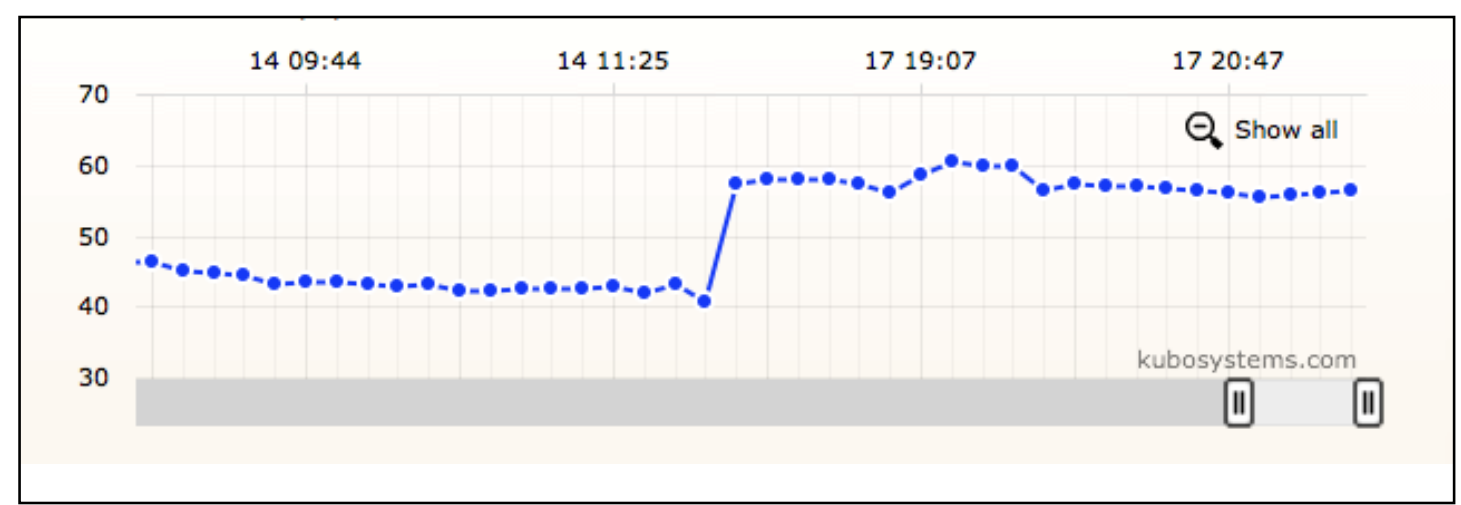

Figure. 7 Relative Humidity Graph (\%)

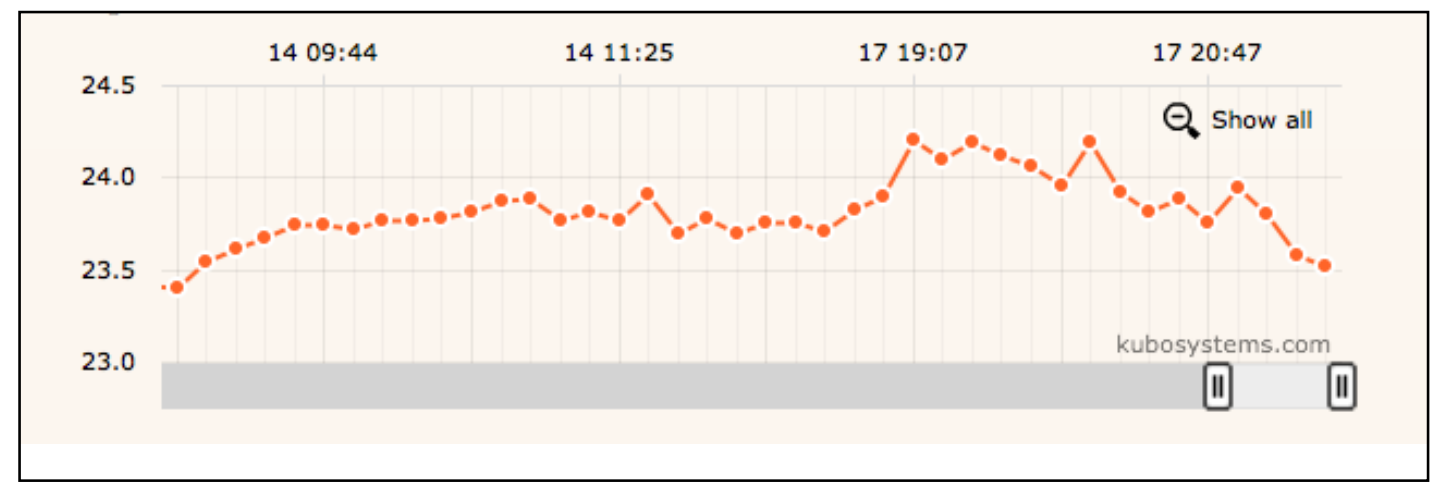

Figure. 8 Temperature Chart $\left({ }^{\circ} \mathrm{C}\right)$

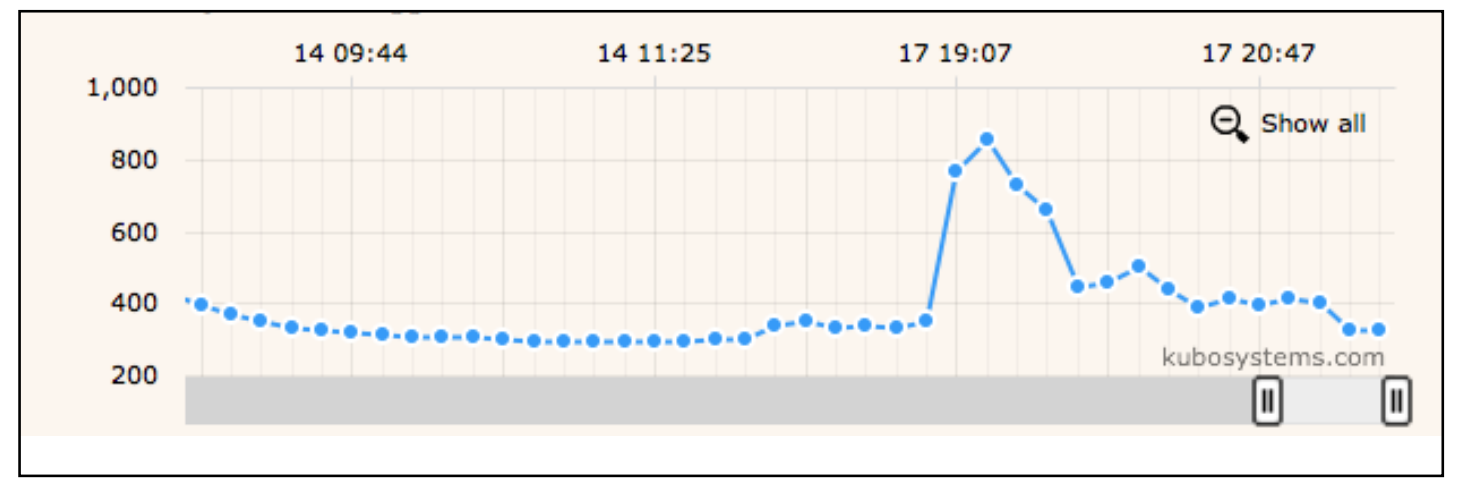

Figure. 9 Graph concentration (ppm) of carbon dioxide (CO2)

The visualization of environmental conditions through graphical figures allows greater perception of the parameter monitored behaviour than the display of numerical tables (Liao, Z.Et al., 2014). On the other hand, the web portal also allows the user to view the historical data in order to provide a more precise analysis of the temporal and detailed development of air quality parameters. Thus, the system is a powerful tool for the detection of air quality problems and decision support for possible interventions in the building.

The iAQ system still in the testing phase. At this stage, the main goal is to make technical improvements, including the proper calibration. Among other advantages, the iAQ system stands out for its modularity, small size, low construction cost, ease of installation and data access through mobile application (Marques G. Et al.,2016). They are planned improvements to the system hardware and software in order to make it more suitable for certain specific purposes, such as use in hospitals, commercial buildings and factories.

\section{CONCLUSION}

This study aimed to present an effective indoor air quality monitoring system which aims to avoid the risk of exposure to contaminants. 
The system was developed using low-cost gas sensors and open source technology such as the Arduino microcontroller development platform. Five sensors for environmental parameters monitoring were used in each iAQ sensor module, but other sensors may be added as needed.

The iAQ system was tested by monitoring two classrooms. The results are very promising, which is a significant contribution to indoor air quality studies. However, the system needs additional experimental validation in real environments, in particular, the assembly of more than two remote modules as was used in laboratory tests in order to ascertain and calibrate the system so increase accuracy.

In addition to system validation, the web portal and mobile application need physical improvements to adapt the system to areas and / or specific problems are planned, such as hospitals, commercial buildings and factories.

Compared to existing systems, the iAQ assumes increased interest due to the use of low cost and open source technologies. Note that the system has advantages in terms of ease of installation and configuration, through the use of wireless technology for communication between the iAQ sensor and iAQ Gateway. However, it is expected further miniaturize the modules through the use of smaller components and more effective methods of heat dissipation. The iAQ also has a mobile Android application, to allow the relevant data view with great versatility.

The system is extremely useful in monitoring the quality conditions of the indoor air and aims to ensure the permanent categorization of indoor air quality, as well as the perception of the behaviour of environmental parameters. Thus, the system can be used to help the building manager to provide more comfortable and productive environments.

\section{REFERENCES}

Spengler JD, Sexton K.: Indoor air pollution: a public health perspective. Science. 221, 9--17 (1983)

USEPA - United States Environmental Protection Agency: Questions About Your Community: Indoor Air, http://www.epa.gov/region1/communities/indoorair.html (2016)

Mukhopadhyay, K., Ramasamy, R., Mukhopadhyay, B., Ghosh, S., Sambandam, S., Balakrishnan, K.: Use of Ventilation-Index in the Development of Exposure Model for Indoor Air Pollution-A Review. Open Journal of Air Pollution. 3, 33-41 (2014)

Pitarma, R., Lourenço, M., Ramos, J.: "Improving ocupational health by modelling indoor pollutant distribution". Facilities (2016)

De Vito, S., Fattoruso,G., Liguoro,R., Oliviero, A., Massera, E., Sansone, C., Casola, V. Di Francia, G.: Cooperative 3D Air Quality Assessment With Wireless Chemical Sensing Networks, Procedia Engineering. 25, pp. 84--87 (2011)

Preethichandra, D.: Design of a smart indoor air quality monitoring wireless sensor network for assisted living. In: conference record - IEEE Instrumentation and Measurements Technology conference (2013)

Yu, T., Lin, C.: An intelligent wireless sensing and control system to improve indoor air quality: monitoring, prediction, and preaction. International Journal of Distributed Sensor Networks (2015)

Al-Haija, Q., Al-Qadeeb, H., Al-Lwaimi, A.: Case Study: Monitoring of AIR quality in King Faisal University using a microcontroller and WSN. Procedia Computer Science. 21, pp. 517-521 ( 2013 )

Preethichandra, D.: Design of a Smart Indoor Air Qiuality Monitoring Wireless Sensor network for Assisted Living. In: IEEE Instrumentation and Measurement Technology Conference (I2MTC2013), pp. 1306_1310. IEEE press, NY (2013)

Yu, T., Lin, C., Chen, C., Lee, W., Tseng, C., Liu, S., Lee, R.: Wireless sensor networks for indoor air quality monitoring, Medical Engineering \& Physics. 35, pp. 231--235 (2013)

Smith, A. (2013). Smartphone ownership-2013 update. Pew Research Center: Washington DC, 12.

Rahmati, A., \& Zhong, L. (2013). Studying smartphone usage: Lessons from a four-month field study. Mobile Computing, IEEE Transactions on, 12(7), 1417-1427.

Alexander J.A.M. van Deursen, Colin L. Bolle, Sabrina M. Hegner, Piet A.M. Kommers, Modeling habitual and addictive smartphone behavior: The role of smartphone usage types, emotional intelligence, social stress, selfregulation, age, and gender, Computers in Human Behavior, Volume 45, April 2015, Pages 411-420, ISSN 07475632, http://dx.doi.org/10.1016/j.chb.2014.12.039.

Hintze, D., Findling, R. D., Scholz, S., \& Mayrhofer, R. (2014, December). Mobile device usage characteristics: The effect of context and form factor on locked and unlocked usage. In Proceedings of the 12th International Conference on Advances in Mobile Computing and Multimedia (pp. 105-114). ACM.

IEEE 802.15 WPAN Task Group4 (TG4), http://www.ieee802.org/15/pub/TG4.html išref(2016)

ZigBee Alliance, http://www.zigbee.org/(2016) iscep:

Digi International Inc., http://www.digi.com/ (2016)

Ramya, V., Palaniappan, B., \& George, B. (2012). Embedded system for automatic irrigation of cardamom field using Xbee-PRO technology. International Journal of Computer Applications, 53(14). 
Michael, M.: Arduino Cookbook. O ReiLLY, USA (2012)

Arduino Website, http://www.arduino.cc (2016)

Datasheet SHT10, http://www.sensirion.com/fileadmin/user_upload/customers/sensirion/ Dokumente/Humidity/Sensirion_Humidity_SHT1x_Datasheet_V5.pdf (2016)

MQ7 Datasheet, https://www.sparkfun.com/datasheets/Sensors/Biometric/MQ-7.pdf (2016)

Telaire T6615 Datasheet, http://www.ge-mcs.com/download/co2-flow/920-474C-LR.pdf (2016)

LDR 5mm Datasheet, https://www.robocore.net/upload/attachments/sensor_ldr_gl5528_145.pdf

Deployment of Wireless Sensor Networks for Air Quality Monitoring, Wang, M., Wang, Y., Li, Q.: Deployment of Wireless Sensor Networks for Air Quality Monitoring, Advanced Materials Research, 712-715, pp. 1851-1855 (2013)

Liao, Z., Peng, Y., Li, Y., Liang, X., Zhao, Y.: A Web-based visual analytics system for air quality monitoring data. In: 22nd International Conference on Geoinformatics (GeoInformatics), pp. 1—6, IEE Press, NY (2014)

Marques, Gonçalo, and Rui Pitarma. "Health informatics for indoor air quality monitoring." Information Systems and Technologies (CISTI), 2016 11th Iberian Conference on. AISTI, 2016. 